\title{
Midbrain tegmental lesions affecting or sparing the pupillary fibres
}

\author{
Naokatsu Saeki, Naohisa Murai, Kenro Sunami
}

\begin{abstract}
Two patients with oculomotor palsy caused by midbrain infarction are reported. In the first, pupillary reaction was affected and in the second this reaction was spared. Because the lesions in the anterior part of the tegmentum were in the upper midbrain in the first patient and in the lower midbrain in the second, it is suggested that the pupillary components of the oculomotor nerve are located in the upper midbrain.
\end{abstract}

(F Neurol Neurosurg Psychiatry 1996;61:401-402)

Keywords: midbrain; oculomotor nerve; pupil sparing

We report the details of two patients with a small midbrain infarction, the first with impairment of pupillary reaction to light and the second in which this reaction was preserved. The aim of this study was to elucidate the topography of oculomotor pupillary fibres in the midbrain tegmentum based on findings using MRI.

\section{Case studies}

CASE 1

A 67 year old man with a 10 year history of hypertension presented with difficulty in opening his left eye on waking up in the morning. On admission several hours after the onset, he had a slight right hemiparesis and hypaesthesia. There was complete left sided ptosis and pronounced limitation of all eye movement except for abduction. His pupils were anisocoric: left $5 \mathrm{~mm}$, right $2 \mathrm{~mm}$. Light reaction was absent on the left side. Brain CT showed a hypodense lesion at the left medial midbrain tegmentum. The patient was diagnosed as having a lacunar infarct causing Weber's syndrome. One week later the right hemiparesis disappeared but the left oculomotor palsy remained.

Two months later, MRI showed a lesion in accordance with the CT findings. Axial T2 weighted images showed a high intensity lesion in the medial cerebral peduncle and tegmentum including the medial red nucleus (fig 1). T1 weighted images in sagittal and coronal sections disclosed a low intensity lesion in the upper midbrain and close to the third ventricle (fig 1 ).

Three months later the oculomotor palsy improved. The patient returned to his previous work after a further three months.

\section{CASE 2}

A 68 year old woman with hypertension for eight years suddenly developed vertigo and
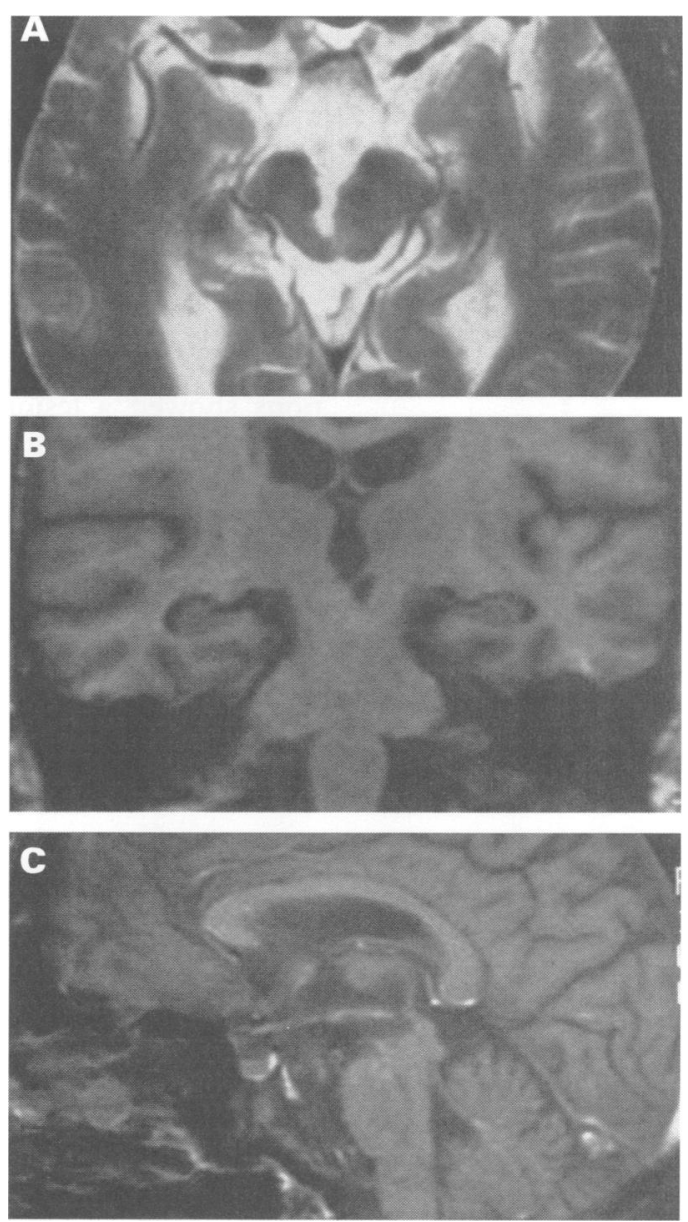

Figure 1 MRI of patient 1. (A) T2 weighted axial image at the level of the red nucleus. A narrow high intensity lesion, $12 \mathrm{~mm}$ in anteroposterior direction, $4 \mathrm{~mm}$ in maximum width, passed through the medial red nucleus. (B) T1 weighted coronal image at the midbrain tegmentum. A low intensity lesion, $6 \mathrm{~mm}$ in height, was noted on the left side close to the midline and immediately below the third ventricle. (C) T1 weighted sagittal image. $A$ narrow low intensity lesion is present at the upper midbrain tegmentum. 
Figure 2 MRI of patient 2. (A) T1 weighted coronal image. A low intensity image. A low intensity lesion $3 \mathrm{~mm}$ in both width and height was noted at the lower midbrain tegmentum (B) T1 weighted sagittal image. The infarcted lesion was located at the caudal midbrain, in striking contrast with patient 1.
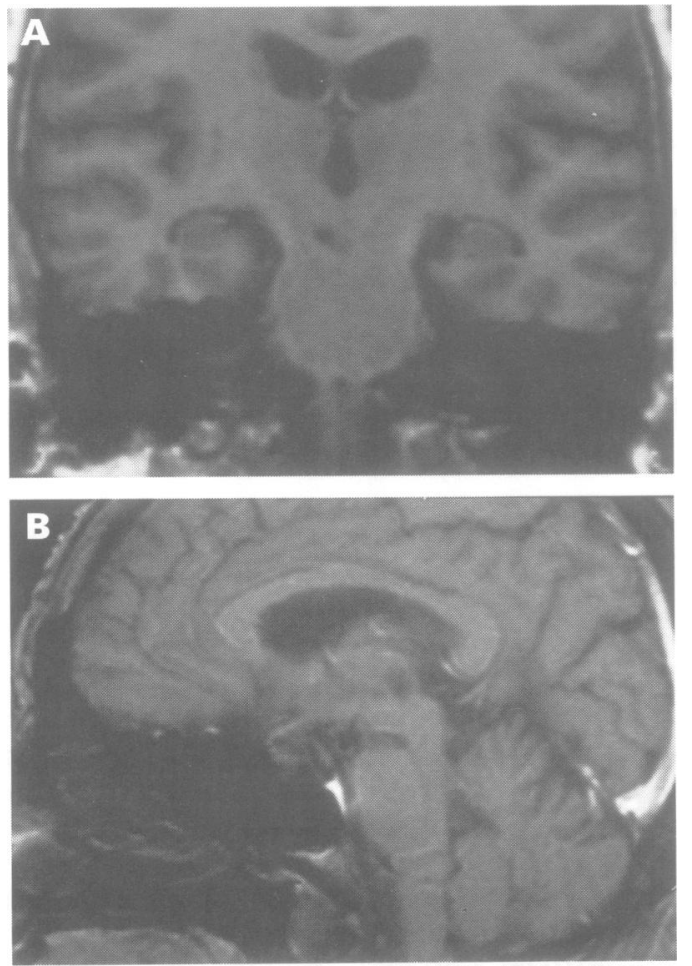

headache. Two hours later, she developed left oculomotor palsy and a slight right hemiparesis. She had ptosis and impairment of all eye movement except abduction. Pupil reaction was normal and no anisocoria was noted. On the next day, the hemiparesis improved and the oculomotor palsy disappeared two weeks later.

One month later, MRI disclosed a low intensity lesion in the medial midbrain tegmentum (fig 2). Coronal and sagittal sections showed a lesion at the lower midbrain and below the red nucleus (fig 2 ).

\section{Discussion}

Two patients with oculomotor palsy caused by midbrain infarction are reported. In the first, pupillary reaction was affected and in the second this reaction was spared.

Because the lesions, in the anterior part of the tegmentum, were in the upper midbrain in the first patient and in the lower midbrain in the second, it is suggested that the pupillary components of the oculomotor nerve are located in the upper midbrain. Such an arrangement is analogous to that of the oculomotor subnuclei, as the visceral nucleus controlling the pupillary fibres is located slightly above the somatic cells controlling the ocular motor muscles and levator palpebrae superioris. This subnuclear arrangement was proposed by Warwick from observations on the rhesus monkey. ${ }^{1}$

Recent advances in MRI have allowed detailed study of the relations between nuclear and fascicular topography and various partial oculomotor palsies of midbrain origin. ${ }^{24}$ However, few MRI reports have focused on the location of pupillary fibres in the midbrain tegmentum. Single cases with a partial oculomotor palsy with or without pupillary signs were recently reported, suggesting the same pupillary arrangement as ours. Although the midbrain lesions in these patients were shown by MRI, the hypothesis concerning the location of pupillary fibres was supported mainly on the basis of neurological combinations of impairment of ocular motion and pupillary midriasis. ${ }^{34}$ Although the suggestion of a rostral location of pupillary fibres in humans is not original, our study is the first to suggest such a location based on MRI evidence in patients with and without pupillary signs.

1 Warwick R. Representation of extra-ocular muscles in oculomotor nuclei of the monkey. F Comp Neurol 1953;98:449-95.

2 Bogousslavsky J, Maeder P, Regli F, Meuli R. Pure midbrain infarction: clinical syndrome, MRI, and etiologic brain infarction: clinical syndrome,

3 Schwarz TH, Lycette CA, Yoon SS, Kargman DE. Clinicoradiographic evidence for oculomotor fascicular Clinicoradiographic evidence for oculomotor

4 Ksiazek SM, Slamovitz TL, Rosen CE, Burde RM, Paris F. Fascicular arrangement in partial oculomotor paresis. Am $\mathcal{f}$ Ophthalmol 1994;118:97-103. 